\section{Applied Physics Technologies}

Incorporated in 1995, Applied Physics Technologies, Inc. was an outgrowth of research conducted for over a decade at Linfield Research Institute (LRI) on transition metal carbides, thermionic, and field electron sources. Demand for products led to the formation of APTech. APTech is working to further advance the science of emission sources for charged particle beam applications.

APTech specializes in thermionic and field emission cathodes. APTech is a specialty producer and supplier of $\mathrm{CeBix}^{\circledR}$ cathodes (cerium hexaboride), $\mathrm{LaB}_{6}$ cathodes (lanthanum hexaboride), HfC cathodes (hafnium carbide), CFE and ESE sources. APTech partners with many end-users, OEMs and government research agencies in various projects involving thermal emission, cold field emission, and refractory metals.

- $\mathrm{CeBix}^{\circledR}$ Cathodes-Cerium Hexaboride $\left(\mathrm{CeBix}^{\circledR}\right)$ Cathodes are the materials of choice for high current cathodes in a variety of advanced and custom applications. APTech is the only producer of $\mathrm{CeBix}^{\circledR}$ cathodes worldwide. Direct replacement for $\mathrm{LaB}_{6}$.

- $\mathrm{LaB}_{6}$ Cathodes-Ideal for many small spot size applications and for high current applications. APTech grows and fabricates our own high quality $\mathrm{LaB}_{6}$ single-crystal material.

- Transition Metal Carbides-Durable electron sources that can perform well in poor vacuum environments. APTech offers single-crystal $\mathrm{HfC}$ and $\mathrm{ZrC}$ transition metal carbide material and others upon request.

- Refractory Metals-We offer single crystal tungsten wire and tungsten rod. Diameters range from $0.12 \mathrm{~mm}$ to $2.5 \mathrm{~mm}$ with orientations of (100) (111) (210) and (310). Other refractory metals upon request.

- R\&D Partnering-Custom cathode design, fabrication, and testing, electron and ion optical modeling, and electron or ion gun production including focusing and deflection. More services available.

- Top Hat Cathodes-Flat emission surface diameters ranging from $1 \mathrm{~mm}$ to $2.8 \mathrm{~mm}$. Ideal for applications requiring high total current in a large beam spot.

- Guard Ring Cathodes-Electron emission defining source configurations. Common form of emission suppression is to embed an oriented single crystal in graphite to suppress side emission and to help shape the electric field.

- Etched Tip Cathodes-Filament mounted, electrochemically etched, field emitters.

- LMIS-Gallium Liquid Metal Ion Sources and (Single Isotope) Gallium 69 LMIS.

- Rebuilding Services-Lower cost option to purchasing a brand-new cathode. Available for some Auger systems, e-beam lithography, cold field emitters, and FEGs. Inquire about your specific model.

All of our products are manufactured in the U.S.A. with distribution channels worldwide. Our markets include microscopy, microanalysis, additive manufacturing, and other industries that use electron sources in their products and work. We primarily work with OEMs to develop and produce cathodes for their unique needs. We also work with government research agencies, colleges and universities, and various research institutes.

\section{AP次TECH}
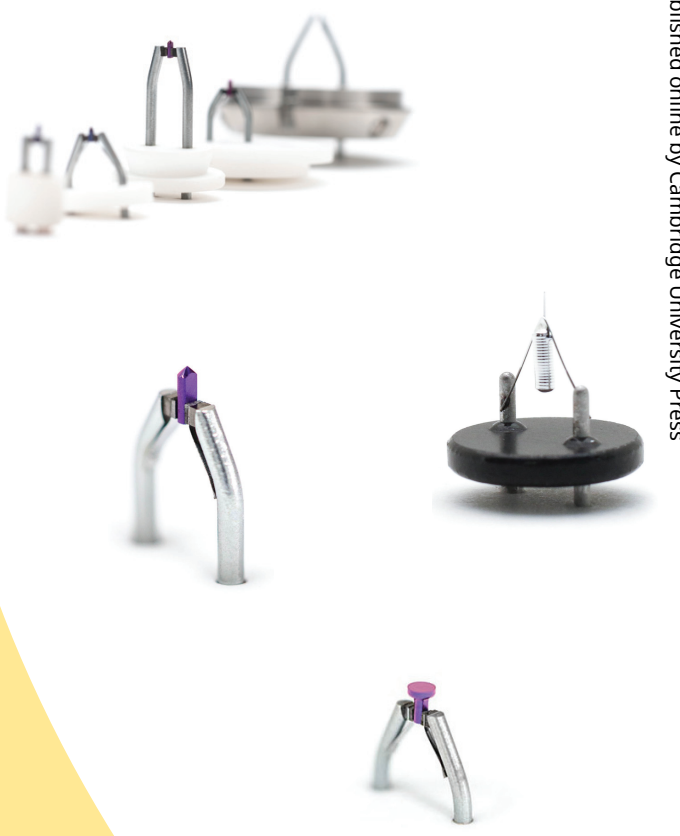

\section{How to find us}

Applied Physics Technologies

1600 NE Miller Street

McMinnville, OR 97128

Tel: +1 (503) 434-5550

Fax: +1 (503) 434-1312

Email: APTsales@a-p-tech.com

www.a-p-tech.com

Our manufacturing facilities and main office are located in McMinnville, Oregon. We have active distributors in the European Union, Taiwan, and South Korea. 